\title{
Self-Pereception of Adults with Intellectual and Developmental Disabilities
}

\author{
Mary Rita Weller \\ Department of Social Work, Kutztown University of Pennsylvania, Kutztown, USA \\ Email: weller@kutztown.edu
}

Received November 25 ${ }^{\text {th }}$, 2013; revised December 25 $5^{\text {th }}$, 2013; accepted January $2^{\text {nd }}, 2014$

\begin{abstract}
Copyright (c) 2014 Mary Rita Weller. This is an open access article distributed under the Creative Commons Attribution License, which permits unrestricted use, distribution, and reproduction in any medium, provided the original work is properly cited. In accordance of the Creative Commons Attribution License all Copyrights $\mathbb{C}$ 2014 are reserved for SCIRP and the owner of the intellectual property Mary Rita Weller. All Copyright @ 2014 are guarded by law and by SCIRP as a guardian.
\end{abstract}

\begin{abstract}
Adults with intellectual and developmental disabilities (IDD) may view themselves as children; indeed they are often treated in a childlike manner by others. Some may develop a self-perception that they are minors and view children more as peers. Using data from this author's (Smith, 2006) unpublished dissertation, developmental self-perception is explored by using a subset of questions from the SSKAAT-R instrument (Griffiths \& Lunsky, 2003). This study discusses how some adults with IDD self-identify as more closely resembling children. A total of 26 adults with IDD, 17 males and nine females, were involved. With IRB approval, questions that would assess understanding regarding perception of self (along with a follow-up question regarding sexual attraction) were posed. Of the 26 participants, $73 \%$ self-identified as more closely resembling adults, whereas the remaining $27 \%$ self-identified as more closely resembling children.
\end{abstract}

Keywords: Self-Perception; Adults with Disabilities; SSKAAT-R; Social Interactions; Role Theory

\section{Introduction}

Adults with intellectual and developmental disabilities (IDD) may view themselves as children; indeed, they are often treated in a childlike manner by others, including family members, agency staff, physicians, and the general public. In turn, having a perception that they are childlike, some adults with IDD may view children more as peers than people that are their chronological age. Some adults with IDD may lack understanding of the difference between their adult self and children which in turn may lead them to have diminished ability to develop meaningful and healthy relationships overall. Moreover, adults that behave in more childlike manners and frequently interact with children on a social level are often scrutinized and stigmatized as having suspicious and questionable behavior which may further impede opportunities for social engagement with other adults. The purpose of this study is to look at the various possibilities that some adults with IDD may self-identify as more closely resembling children.

The notion that some adults with IDD perceive themselves as children due to how others treat and perceive them is reported in the literature. Evans, McGuire, Healy, and Carley (2009) assessed the attitudes of staff and family regarding the sexuality of people with IDD. Evans, et al. (2009) indicated how relationships may play a significant role in behaviors that people engaged in regardless of disability status. Siebelink, de Jong, Taal, and Roelvink (2006) discussed how people characterized social interactions based on expected and actual differences in roles. Attractions and sexual experiences are not simply driven by biological forces, but also role expectations that are socially constructed (2006). Thus the question investigated is: "Do some adults with IDD perceive themselves as more closely resembling children?”

\section{Theoretical Perspective}

Role theory provides the theoretical basis for this study in regards to self-perception. According to role theory, individuals develop their identity and understanding of their selves based on the roles that they play within their culture. In 1934, "George Herbert Mead, the sociologist, introduced the concept of role taking and focused on its significance in the development of an individual's self-concept” (Davis, 1986: p. 542). "We lay the foundation for a sense of who we are by identifying with significant others, internalizing their attitudes as our own, and seeing ourselves as others see us” (1986: p. 542). Since individuals with IDD may be treated similar to children by their families, support persons, physicians, educators, and general society members, then according to role theory these individuals may develop the self-concept of being a child as they take on the role expectation. "Role expectations are the set of expectations for the behaviors of a person or a position held by a particular person or by a generalized other" (1986: p. 545).

On another take of the role theory, Simon and Gagnon (2003) explored the correlation between sexual knowledge and attitudes and between attitudes and experiences as they developed their theory on sexual scripts which addresses the origins, influences and changes of how people develop their sexual behaviors and understanding. Simon and Gagnon's study (2003) provides additional insight about the theoretical framework that 
is presented in this study in regards to role theory in their interpretation of sexual scripts. Individuals with IDD are influenced by roles that they observe and fulfill them just like everyone else. Part of the study is exploring the insight that individuals with IDD have about themselves and their developmental stage.

Smith and Willner (2004) examined the relationship paradigms of men with IDD and how stronger, healthier relationships may be a deterrent in men with IDD from acting out violent and aggressive sexual behavior. Steptoe, Lindsay, Forrest, and Power (2006) correlated poor social adjustments and relationships with acting out of violent, aggressive sexual behavior of sex offenders with IDD. It should be noted that this current analysis does not investigate how self-identity may influence sexual behavior, only looks at how adults with IDD may perceive themselves either as children or adults.

In looking at this current investigation, it also raises questions about how self-concept is developed overall. When and how does the concept of self emerge? Is there a physiological difference between how people with cognitive disabilities and those without any disability develop their own self-concept or is the development of self-concept solely dependent on the social environment or is it a combination of both?

Erikson (1982) noted the struggle, at the time of transition from adolescence to young adulthood and eventually the passage into full adulthood, is between generativity versus selfabsorption and stagnation. "Generativity encompasses procreativity, productivity, and creativity, and thus the generation of new beings as well as of new products and new ideas, including a kind of self-generation concerned with further identity development” (1982: p. 67). Essentially, Erikson notes that the stage of adulthood takes on a new commitment towards caring for others, which often corresponds with the time of procreation. Ironically, most adults with IDD are being taken care of rather than moving into the adult role of providing care for others; this may account for the possibility of why some adults with IDD do not necessarily relate to the role of an adult and/or acquire the adult self-identity.

Although Erikson's work may be considered to be somewhat flawed and outdated, it is important to note his work around human development and how it may be used to provide some understanding of how individuals transition from one stage of life development into the next. Erikson (1982) theorized about the development of individuals as they progressed through various life stages starting at infancy through old age. He (Erikson, 1982) broke down human development into eight epigenetic orders (infancy, early childhood, play age, school age, adolescence, young adulthood, adulthood, and old age) with eight corresponding stages (psychosexual, psychosocial crises, radius of significant relations, basic strengths, basic antipathies, social order, binding ritualization, and ritualism). "A pervasive sense of identity brings into gradual accord the variety of changing self-images that have been experienced during childhood (and, that, during adolescence, can be dramatically recapitulated) and the role opportunities offering themselves to young persons for selection and commitment. On the other hand, a lasting sense of self cannot exist without a continuous experience of a conscious 'I,' which is the numinous center of existence: a kind of existential identity, then which must gradually transcend the psychosocial one" (1982: p. 73). "In summary, the process of identity formation emerges as an evolving configuration - a configuration that gradually integrates constitutional givens, idiosyncratic libidinal needs, favored capacities, significant identifications, effective defenses, successful sublimations, and consistent roles" (1982: p. 74). Taking into consideration all of these processes as delineated by Erikson (1982), one can only wonder how $73 \%$ of the participants in this study managed to develop any sense of self as adults when considering their need for ongoing support and in some cases close supervision. As noted previously, adults with IDD are often treated like children as they often need assistance with basic daily life skills including personal care, money management, supervision, and so forth.

Piaget's work also has been criticized and debated through the years, but again his classic theories regarding cognitive development may provide some understanding into how individuals develop self-perceptions. Fischer (1980) was heavily influenced by Piaget research as he also explored the construction of cognitive development. Fischer (1980) noted that little research exists related to cognitive development beyond adolescence. Fischer focuses on Piaget's framework of cognitive development that virtually ends with formal operations: "Adolescents entering the formal operational period have achieved fully logical thinking, and there is little more for them to do, except perhaps to extend their logical thinking to new context areas” (1980: p. 496). Piaget (1972) also took into consideration that "the speed of development can vary from one individual to another and also from one social environment to another; consequently, we may find some children who advance quickly or others who are backward, but this does not change the order of succession of the stages through which they pass" (p. 41). Piaget (1972) also noted changes in adolescents' social point of view diverging from childhood. Here they begin to develop their ability to hypothetical reasoning; "hence, the adolescent's capacity to understand and even construct theories and to participate in society and the ideologies of adults” (1972: p. 42). One may conceptualize that since individuals develop at different stages, it may be that some individuals with IDD successfully transitioned into the adolescent stage and eventually adulthood, whereas, other individuals with IDD may require a much longer period of time to acquire the cognitive abilities in adolescence and eventually adulthood, while other individuals with IDD may never transition cognitively beyond childhood.

Piaget (1972) also compared development speed of children based on their environment. "These different speeds would be due to the quality and frequency of intellectual stimulation received from adults or obtained from the possibilities available to the children for spontaneous activity in their environment” (1972: p. 44). He proposed that poor stimulation and activity, as well as extremely disadvantaged conditions could result in slowed down development (1972). Piaget looked at situations that included individuals with normal development and failed to include individuals with any disabilities. Additionally, Piaget (1972) noted that "the study of young adults is much more difficult than the study of the young child as they are less creative, and already part of an organized society that not only limits them and slows them down but sometimes even rouses them to revolt” (p. 47). Taking into consideration all of the challenges in studying adults, layer on top of that developmental disabilities, and it almost seems impossible to determine the understanding of why some individuals with IDD view themselves accurately as adults, while others do not.

This study predominately focuses on self-perception of adults with IDD but also takes into consideration aspects of sexual attraction and behavior. Some related sexuality theories 
regarding sexual attraction and behavior include evolutionary psychological, social learning, and schema. Evolutionary psychological theory is based from Darwin's theory of evolution from natural selection. "According to evolutionary psychological theory, the physical features that people find attractive are precisely those that have been reliably correlated with reproductive success throughout human existence" (Wagstaff, Abramson, \& Pinkerton, 2000, p. 11). One can only assume that according to the evolutionary psychological theory individuals with visible disabilities would be considered sexually undesirable as they may not exude the ideal state of youth and healthfulness. "Symons (1995) argues that the appearance of youth and healthfulness is universally associated with female attractiveness because these qualities act as de facto markers for reproductive fitness" (p. 81). "Consequently, he predicts that in all societies in which women attempt to alter their appearance through cosmetics, diet, exercise, and dress - they will do so in a manner that accentuates the appearance of youth and health" (1995: p. 82). "Conversely, men are assumed to have inborn mechanisms for detecting these qualities in women” (1995: p. 83). In considering this theory in relation to adults with IDD, this may be where the misconception that adults with disabilities are non-sexual beings or at least that they should be since many will have obvious physical features related to their disability. Even if the individual does not have an apparent visible disability, once they verbally communicate, their disability often is obvious due to grappled speech and/or cognitive emissions.

Social learning theory provides additional accepted principles regarding sexual behavior and relationships. Social learning theory is based on the work of Kurt Lewin (1935) and Julian Rotter (1954), and Albert Bandura (1977, 1986). "It states that reinforcements that occur in society shape attitudes and behaviors” (Hyde \& DeLamater, 2000: p. 34). If an individual receives positive reinforcement for a behavior, than that individual will most likely continue to engage in the same behavior; this is often referred to as positive reinforcement or conditioning. Social learning theory assumes that individuals learn by modeling behavior of other people. Hyde and DeLamater (2000) discuss how social learning theory is "useful in explaining the development of gender identity, or one's sense of maleness or femaleness” (p. 34). The examples often used in relationship to social learning theory is how little girls may imitate their mother's by playing grownup by dressing up in their mother's clothing and putting on makeup. Adults with IDD learn by imitation and modeling behavior that they see in their environments and through media. Social learning theory can be connected back to how adults with IDD develop concepts related to roles of adults and children; if they are treated like adults and have responsibilities and decision making expectations, according to the social learning theory, they will model adult behavior. In turn, when adults with IDD are treated similar to children by others in their environment, they may take on the role and behavior expectations of children such as dependency and lack of autonomy.

Schema theory is also relevant for this study as it looks at gender role development, essentially how genders differentiate. "Psychologist Sandra Bem (1981) has proposed a schema theory to explain gender-role development and the impact of gender on people's daily lives and thinking” (p. 354). "Schema" is a term taken from cognitive psychology. "A schema is a cognitive structure, a network of associations that organizes and guides an individual's perception” (1981: p. 355). “As children learn the contents of the society's gender schema, they learn which attributes are to be linked with their own sex and, hence, with themselves” (1981: p. 355). Bem (1981) notes that everyone possesses a gender schema - the set of attributes (behaviors, personality, appearance) that we associate with males and females. Our gender schema, according to Bem (1981), predisposes us to process information on the basis of gender. All people basically categorize behavior based on gender. Take for example, upon hearing the news that a woman is pregnant, one of the first inquiries is often does she know if it is a boy or a girl. There are social expectations associated with gender behavior based on each person's culture.

\section{Study Design/Method}

The data being analyzed for this study is a portion of that collected for this author's unpublished doctoral dissertation (Smith, 2006). A mixed methods, non-experimental design was chosen in order to minimize potential communication obstacles and to fully explore the views of the participants. The qualitative approach included face-to-face interviews in which three questions related to self-identity and attraction were presented verbally to the participants, meeting the criteria for qualitative research (Bogdan \& Biklen, 1992):

1) Has the natural setting as the direct source of data and the researcher is the key instrument;

2) Is descriptive as the data collected are in the form of words or pictures rather than numbers;

3) Concerned with process rather than simply outcomes or products;

4) Tends to analyze data inductively;

5) "Meaning" is of essential concern to the approach and interested in the ways different people make sense of their lives. (p. 30)

The SSKAAT-R instrument (Griffiths \& Lunsky, 2003) was used to assess the participants regarding their overall understanding about relationships and sexuality. SSKAAT-R (2003) contains a total of 370 questions designed to assist educators and clinicians working with persons with developmental disabilities. It evaluates information persons with developmental disabilities have about their bodies, socio-sexual intimacy, relationships and issues of abuse. This tool was selected for implementation because of its relevant assessment themes, including: overall understanding about relationships, bodies and sexuality; women's knowledge of men's bodies (for women only) and men's bodies (for men only); intimacy, pregnancy, childbirth, and childrearing; birth control and sexually transmitted infections; and healthy sexuality and boundaries. For the purposes of this line of inquiry, three questions were analyzed. This subset of questions from the SSKAAT-R (2003) asks individuals to identify the person that most resembles them from a series of photos that are displayed on one sheet; the four photos represent one male adult, one female adult, one male child, and one female child. Specifically, the item being analyzed is "show me the picture of the person who is most like you". This study also takes into account the two related questions posed with this initial question regarding "person most like you" which are "show me the people it is okay for you to go out with on a romantic date and show me the people with whom it is okay for you to have sex" (2003: p. 77). These queries are relevant to self-identity and attraction; the face-to-face interviews 
contribute to the expression of those complex factors.

Requirements for participation were that the individuals had to be over the age of 18 and have a diagnosis of IDD ranging from mild to moderate. The 26 participants were between the ages of 21 to 57 years old $(\mathrm{M}=38, \mathrm{SD}=11.56)$ with the diagnosis of mild to moderate IDD. These individuals were referred for evaluation due to their challenges related to relationships and/or sexuality issues. The heightened risk for this population required a full review for Institutional Review Board (IRB) approval; all human subject guidelines were followed. It was determined that their special status as referred clients did not disqualify them for this study; indeed, it was an opportunity to examine the link between documented behavior and self-concept. Consent was secured both from the individuals and their family members. Information used for the current analysis includes gender, age, and responses to three questions that used photos portraying an adult male, adult female, child male, and child female.

\section{Results}

After the text edit has been completed, the paper is ready for the template. Duplicate the template file by using the Save As command, and use the naming convention prescribed by your journal for the name of your paper. In this newly created file, highlight all of the contents and import your prepared text file. You are now ready to style your paper. Four out of the 17 males self-identified as most closely resembling the male child; 12 males aligned themselves with the adult male. One male selfidentified as most closely resembling the female child as he stated that, "she dresses like me." Two out of nine females indicated that they most resembled the female child; whereas, the seven other females stated that they most resembled the female adult. One of the females that indicated that she most resembled the female child also indicated that it would be okay to date and/or have sex with the female child. Two of the males that stated that they most resembled the male child, also indicated their attraction to the female child. Another male that identified himself most closely resembling a male child indicated his attraction towards the male child. Although another male that identified himself most resembling a male adult indicated his attraction towards the female child. Out of the nine females in the total sample, one stated that it would be okay to date a male child, but have sex with a male adult; the other eight female participants either indicated that it was okay to date and/or have sex with an adult, or neither behavior was okay for them to engage in. Ten out of the 17 male participants stated that it would be okay to date and/or have sex with an adult, or neither behavior was okay for them to engage in. As stated earlier, an interesting discovery in the original study (Smith, 2006) was that seven (27\%) out of the 26 participants choose the photo representing a child, whereas, the other 19 (73\%) participants accurately choose the adult photo.

The data in the graph (Figure 1) represents total number of participants in the study and their correlating responses to the question: "show me the picture of the person who is most like you." The first graph shows total number of participants $(\mathrm{N}=$ $26)$ with the breakdown of men $(\mathrm{N}=17)$ and females $(\mathrm{N}=9)$ with their responses as pointing to the adult photos (man or woman) $(\mathrm{N}=19)$ or child photos (boy or girl) $(\mathrm{N}=7)$. The second graph represents the males' responses $(\mathrm{N}=17)$ to the question of "show me the picture of the person who is most like

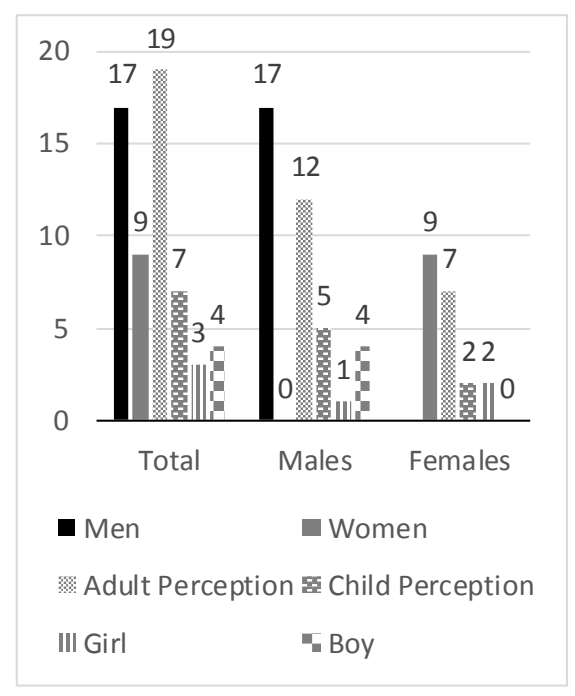

Figure 1.

Self-Perception Responses: Who is most like you?

you." Out of the 17 men in the study, 12 pointed to the adult photos; five pointed to the photo of a child (boy or girl): four of the males pointed to the photo of the boy, whereas, one male pointed to the photo of the girl. The third graph represents the responses of the nine women in the study. Seven of the women pointed to an adult photo in response to this question, whereas, two women pointed to the girl's photo.

\section{Discussion}

The question investigated was: "Do some adults with IDD perceive themselves as more closely resembling children?” There may be numerous reasons why seven out of the 26 individuals indicated their resemblance to children when given the four choices between adult male, adult female, child male, and child female. Classic theorists such as Piaget and Erikson would most likely posit that due to the cognitive disability status of these individuals, some of their developmental transitions were disrupted and not fully realized because of a combination of physiological and social conditions present in their environments. This theoretical premise, however, does not explain the variation amongst the other 19 individuals that accurately pick an adult representation for themselves.

As noted, one male who self-identified as most closely resembling the female child as he stated that "she dressed like me." Based on this one response, it may be further understood that some and/or all individuals interpreted the questions differently than what the original purpose was from the investigator's stance. Essentially, the question may have been misunderstood and the person may have thought they were being asked which person dresses most like you? In the photos displayed from the SSKAAT-R (Griffiths \& Lunsky, 2003) tool, none of the males and/or females are overtly masculine or feminine, and in all four photos everyone wore a basic pullover shirt or sweater or sweatshirt and pants; the man displayed had an obvious mustache and the woman was the only one with a graphic line design on her sweater. It should also be noted that all photos were in black and white therefore, colors were not apparent.

The questions 1) "show me the picture of the person who is most like you"; 2) "show me the people it is okay for you to go 
out with on a romantic date"; 3) "show me the people with whom it is okay for you to have sex" as well elicited mixed responses. Simon and Gagnon (2003), well known for their development of the idea of sexual scripts, may interpret feedback from the individuals that answered questions in regards to going on a romantic date and/or okay to have sex with individuals that may be playing along, and answering the questions based on the individuals' understanding of their dating and sexual roles in our culture. The individuals that responded to the questions that neither dating and/or having sex with another individual portrayed in the photo, may be responding to sexual scripts that essentially support the idea of abstinence for adults with IDD. Messages of being non-sexual are constantly being reinforced to individuals with IDD through their interactions with family, peers, media, physicians, staff, etc. Additionally, the individuals may have mistaken the hypothetical stance of the questions being posed, such as looking at these individuals as being strangers or perhaps not being sexually attractive or appealing to them. As for the six individuals, two females and four males, that answered positively in regards to either dating and/or having sex with the children, they too may have misunderstood the line of questioning in that they may not have even known what was meant by dating and/or having sex; it should be noted that the line of questioning was direct and uniform with little or no improvisation.

Overall, this current study proposed to address some of the reasons that seven adults with IDD indicated their resemblance to children, in contrast to the other 19 adults with IDD that accurately indicated their resemblance to adults. One must caution that this sample is too small to be a true representation of feedback from adults with IDD. There were 26 people in this study; in United States alone there are over six million people that may have the diagnosis of IDD as two percent of the population is deemed to have IDD (U.S. Department of Commerce, 2013). These 26 individuals may not be a fair representation of adults with IDD. The data were collected from individuals that were referred for evaluation due to challenges related to relationships and/or sexuality issues; therefore, they may overrepresent individuals with IDD that may demonstrate the selfperception of a child and/or an attraction towards children.

A further understanding about how adults with IDD may maintain a misunderstanding about their self-identity may lead to quality of life issues. There continue to be many struggles in supporting adults with IDD in securing basic human rights related to autonomy and self-determination. Field and Hoffman (1994) define self-determination as "the ability to identify and achieve goals based on a foundation of knowing and valuing oneself” (p. 164). "The individual knowledge, skills, and beliefs that lead to self-determination are delineated according to five components: know yourself, value yourself, plan, act and experience outcomes, and learn" (Field \& Hoffman, 2002: p. 113). Schools are mandated by the Individuals with Disabilities Education Act (IDEA) to support students with disabilities that identify and develop practices and programs that support selfdetermination for youths with disabilities (Ward \& Kohler, 1996). Essentially, adults with IDD need to be encouraged and supported to make their own decisions and choices about their lives which is the emphasis of self-determination. It is important for them to view themselves physically, mentally, and socially as their adult self, rather than as children that are dependent and often void of any decision making ability. There is both an empirical and theoretical link between self-determina- tion and quality of life (Wehmeyer \& Schalock, 2001).

Based on historical and current treatment of adults with IDD, it should not be surprising that some adults with IDD more closely align themselves with children. "For many persons with intellectual and developmental disabilities, it is during their adolescent years that they begin to recognize differences between themselves and their typically developing peers” (Jones, 2012: p. 31); this recognition may lead to feelings of inadequacy, frustration, or isolation (Evans, 1998). Moreover, how one views the self is critical to one's long-term personal development (Harter, 1986). It should not be surprising that once individuals with IDD reach adulthood, often they do not perceive themselves as adults as they continue to rely on others for basic support such as personal care, finances, housing, and essentially everyday living needs. It would be interesting to learn more about each individual in how they interact with others in their environment. How much autonomy do individuals have within their social environments? What are the relationship dynamics within their families and vocational environments? Those 19 participants that accurately perceived themselves as adults may actually be encouraged to be more independent and provided with the support to be more successful as autonomous adults.

Future research should include a more widespread assessment of adults with IDD that includes individuals without problematic relationship and sexuality issues. Additional research should include their perception around the concepts of dating, sexuality, and adulthood. As noted earlier, one individual self-identified with a child in the photo based on similarity in their clothing; in this case, it may be that the individual did not truly comprehend the question posed and simply chose the child due to clothing rather than developmental age differences. Additionally, when doing any form of intervention and/or research with individuals that have IDD or any other form of cognitive disability, it is important to first learn what their understanding of words and/or concepts is.

This research demonstrates one step towards opening up the conversation with adults with IDD to further engage them in thinking about their self-perception and identity in terms of their relationships, including relationships that are intimate and also their understanding about human development. Self-perception is an important piece to successfully transition into adulthood in terms of overall human development: social, psychological, and biological development. Relationship building hinges on individuals' abilities to accept growth, as well as explore beyond themselves by connecting with others.

\section{Acknowledgements}

Thanks to Dr. Sharon Lyter for her oversight and assistance.

\section{REFERENCES}

Badura, A. (1986). Human agency in social cognitive theory. American Psychologist, 37, 1175-1182.

Badura, A. (1977). Social learning theory. Englewood Cliffs, NJ: Prentice Hall.

Bem, S. (1981). Gender schema theory: A cognitive account of sex typing. Psychological Review, 88, 354-364.

http://dx.doi.org/10.1037/0033-295X.88.4.354

Bogdan, R. C., \& Biklen, S. K. (1992). Qualitative research in education $\left(2^{\text {nd }}\right.$ Ed.). Boston: Allyn \& Bacon.

Bruess, C. E., \& Greenberg, J. S. (1994). Sexuality education: Theory and Practice ( $3^{\text {rd }}$ Ed.). Madison, WI: WCB Brown \& Benchmark, Publishers. 
Davis, L. V. (1986). Social work treatment: Interlocking theoretical approaches ( $3^{\text {rd }}$ Ed.). New York: The Free Press.

Erikson, E. (1982). The life cycle completed. New York: W. W. Norton \& Company.

Evans, D. S., McGuire, B. E., Healy, E., \& Carley, S. N. (2009). Sexuality and personal relationships for people with intellectual disability. Part II: Staff and family care perspectives. Journal of Intellectual Disability Research, 53, 913-921. http://dx.doi.org/10.1111/j.1365-2788.2009.01202.x

Evans, D. W. (1998). Development of the self-concept in children with mental retardation: Organismic and contextual factors. In J. A. Burack, R. M. Hodapp, \& E. Zigler (Eds.), Handbook of mental retardation and development. New York: Cambridge University Press.

Field, S., \& Hoffman, A. (1994). Development of a model for selfdetermination. Career Development for Exceptional Individuals, 17, 159-169. http://dx.doi.org/10.1177/088572889401700205

Field, S., \& Hoffman, A. (2002). Preparing youth to exercise self-determination: Quality indicators of school environments that promote the acquisition of knowledge, skills, and beliefs related to self-determination. Journal of Disability Policy Studies, 13, 113-118. http://dx.doi.org/10.1177/10442073020130020701

Fischer, K. W. (1980). A theory of cognitive development: The control and construction of hierarchies of skills. Psychological Review, 87, 477-531. http://dx.doi.org/10.1037/0033-295X.87.6.477

Griffiths, D. M., \& Lunsky, Y. (2003). Socio-sexual knowledge and attitudes assessment tool-revised. Wood Dale. IL: Stoelting Co.

Harter, S. (1986). Cognitive-developmental processes in the integration of concepts about emotions and the self. Social Cognition, 4, 119151. http://dx.doi.org/10.1521/soco.1986.4.2.119

Hyde, J. S., \& DeLamater, J. D. (2000). Understanding human sexuality $\left(7^{\text {th }}\right.$ Ed.). Boston: McGraw Hill.

Jones, J. (2012). Factors associated with self-concept: Adolescents with intellectual and developmental disabilities share their perspectives. American Association on Intellectual and Developmental Disabilities, 50, 31-40. http://dx.doi.org/10.1352/1934-9556-50.1.31

Lewin, K. (1935). A dynamic theory of personality. New York: McGraw Hill.

Piaget, J. (1972). Intellectual evolution from adolescence to adulthood. Human Development, 15, 40-47.

Rotter, J. (1954). Social learning and clinical psychology. Englewood
Cliffs, NJ: Prentice Hall. http://dx.doi.org/10.1037/10788-000

Siebelink, E. M., de Jong, M., Taal, E., \& Roelvink, L. (2006). Sexuality and people with intellectual disabilities: Assessment of knowledge, attitudes, experiences, and needs. Mental Retardation, 44, 283-294.

http://dx.doi.org/10.1352/0047-6765(2006)44[283:SAPWID]2.0.CO; $\underline{2}$

Simon, W., \& Gagnon, J. (2003). Sexual scripts: Origins, influences and changes. Qualitative Sociology, 26, 491-497. http://dx.doi.org/10.1023/B:QUAS.0000005053.99846.e5

Smith, M. R. (2006). Providing relationship building blocks for adults with intellectual disability. Unpublished PhD Dissertation, Chester, PA: Widener University.

Smith, M., \& Willner, P. (2004). Psychological factors in risk assessment and management of inappropriate sexual behaviour by men with intellectual disabilities. Journal of Applied Research in Intellectual Disabilities, 17, 285-297. http://dx.doi.org/10.1111/j.1468-3148.2004.00210.x

Steptoe, L., Lindsay, W. R., Forrest, D., \& Power, M. (2006). Quality of life and relationships in sex offenders with intellectual disability. Journal of Intellectual and Developmental Disability, 31, 13-19. http://dx.doi.org/10.1080/13668250500488652

Symons, D. (1995). Beauty is the adaptation of the beholder: The evolutionary psychology of human female attractiveness. In P. R. Abramson, \& S. D. Pinkerton (Eds.), Sexual nature/sexual culture (pp. 80-118). Chicago: University of Chicago Press.

United States Department of Commerce (2013). United States Census Bureau. www.census.gov

Wagstaff, D. A., Abramson, P. R., \& Pinkerton, S. D. (2000). Research in human sexuality. In L. T. Szuchman, \& F. Muscarella (Eds.), Psychological perspectives on human sexuality (pp. 3-59). New York: John Wiley \& Sons, Inc.

Ward, M., \& Kohler, P. D. (1996). Teaching self-determination: Content and process. In L. E. Powers, G. H. S. Singer, \& J. Sowers (Eds.), Promoting self-competence in children and youth with disabilities: On the road to autonomy (pp. 275-322). Baltimore: Brookes.

Wehmeyer, M. L., \& Schalock, R. (2001). Self-determination and quality of life: Implications for special education services and supports. Focus on Exceptional Children, 33, 1-16. 